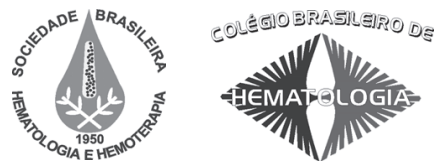

\title{
Densidade mineral óssea em crianças talassêmicas: uma experiência brasileira
}

\section{Bone mineral density in thalassemic children: a Brazilian experience}

Perla Vicari ${ }^{1}$

Margarida M. P. Correal

Vera L. Szejnfeld ${ }^{2}$

Maria Stella Figueiredo

Rita de Cássia R. Cavalheiro ${ }^{4}$

Mihoko Yamamoto ${ }^{5}$
A osteoporose, caracterizada por aumento da fragilidade óssea e suscetibilidade a fraturas, é inversamente proporcional ao pico de massa óssea adquirido na infância. Por outro lado, a doença óssea é uma importante causa de morbidade em pacientes portadores de beta-talassemia maior (TM). Apesar de intensamente descrita em pacientes talassêmicos adultos, não existem estudos sobre as alterações de densidade óssea em crianças talassêmicas brasileiras. Foram avaliados 11 pacientes (idade mediana de 10,0, variando de 5 a 12 anos), portadores de TM, e 24 crianças (idade mediana de 9,5, variando de 6 a 12 anos) saudáveis, utilizando medida de emissão dupla de raios-X para avaliar a densidade mineral óssea (DMO). A análise de marcadores bioquímicos tais como concentração de ferritina sérica, cálcio ionizado, fosfatase alcalina, fósforo, albumina, tempo de protrombina e fator $V$ foi realizada. A estatura foi significativamente diferente entre os dois grupos estudados, $p<0,05$. Os pacientes talassêmicos mostraram valores significativamente inferiores de DMO (mediana 0,61 $\mathrm{g} / \mathrm{cm}^{2}$ ) quando comparados aos indivíduos controles (mediana $0,69 \mathrm{~g} / \mathrm{cm}^{2}$ ), $p<0,05$. A relevante perda óssea encontrada na maioria das crianças talassêmicas estudadas reforça a necessidade de identificação e tratamento adequado da osteopenia, reduzindo a morbidade destes indivíduos. Este é o primeiro estudo, descrito na literatura, que avalia a DMO em crianças talassêmicas brasileiras. Rev. Bras. Hematol. Hemoter. 2008;30(6):445-448.

Palavras-chave: Densidade mineral óssea; talassemia; osteopenia; densitometria; anemia.

\section{Introdução}

Osteoporose é uma doença metabólica óssea sistêmica, caracterizada pela diminuição da densidade mineral óssea e deterioração da microarquitetura do tecido ósseo, com consequente aumento da fragilidade e suscetibilidade a fraturas ósseas. É mais frequente no sexo feminino após a menopausa, sendo que, após os 50 anos, cerca de $30 \%$ das mulheres e $13 \%$ dos homens apresentam fraturas. ${ }^{1,2}$ Entretanto, a osteoporose e a osteopenia atualmente são alvos de estudos em pacientes pediátricos, visto que é reconhecido que a densidade mineral óssea (DMO) de indivíduos adultos depende do pico de massa óssea adquirido até o final da segunda década de vida. ${ }^{1-3}$

Adicionalmente, a osteoporose pode ser instalada na infância e adolescência em decorrência de complicações de

${ }^{1}$ Médica da Disciplina de Hematologia e Hemoterapia Unifesp-EPM - São Paulo-SP.

${ }^{2}$ Docente da disciplina de Reumatologia da Universidade Federal de São Paulo - Unifesp-EPM - São Paulo-SP.

${ }^{3}$ Professora Adjunta da Disciplina de Hematologia e Hemoterapia - Unifesp-EPM - São Paulo-SP.

${ }^{4}$ Residente da Universidade Federal de São Paulo - Unifesp-EPM - São Paulo-SP.

${ }^{5}$ Professora Adjunta da Disciplina de Hematologia e Hemoterapia - Unifesp-EPM - São Paulo-SP.

Disciplinas de Hematologia e Hemoterapia e Reumatologia, Universidade Federal de São Paulo - Escola Paulista de Medicina (Unifesp-EPM), São Paulo-SP.

Correspondência: Perla Vicari

Universidade Federal de São Paulo - Escola Paulista de Medicina (Unifesp-EPM)

Disciplina de Hematologia e Hemoterapia

Rua Botucatu, 740 / $3^{\circ}$ andar - Vila Mariana

04023-000 - São Paulo-SP - Brasil

Tel. (+55 11) 5576-4240; Fax. (+55 11) 5571-8806

Email: perlavicari@hemato.epm.br 
doenças crônicas, tais como: patologias intestinais, renais ou hepatobiliares, fibrose cística, anorexia, artrite reumatóide ou decorrente de algumas terapêuticas como metotrexate e corticóides. ${ }^{3}$

A talassemia maior (TM) é uma anemia hereditária, potencialmente letal na infância, caracterizada por anemia grave, esplenomegalia e aparência mongolóide característica. Estes pacientes apresentam também outras manifestações ósseas, tais como: dores, redução da densidade óssea, retardo de crescimento e da idade óssea, escoliose, compressões medulares, fraturas patológicas, osteopenia e osteoporose. $^{4}$

O tratamento da TM, transfusões crônicas e quelação do ferro têm melhorado a expectativa e qualidade de vida destes pacientes. No entanto, apesar desta terapêutica, as alterações ósseas ainda são observadas e consideradas importante causa de morbidade nestes indivíduos. ${ }^{4}$

As alterações ósseas parecem ser secundárias à expansão medular e adelgaçamento cortical, entretanto sua etiologia ainda é bastante controversa. ${ }^{5}$ Vários fatores podem afetar o metabolismo e o turnover ósseo destes pacientes, tais como alterações hormonais, deficiência vitamínica, sobrecarga de ferro e a própria terapia quelante. ${ }^{6-8}$ Apesar de intensamente descrita em pacientes talassêmicos adultos, não existem estudos sobre as alterações de densidade óssea em crianças talassêmicas brasileiras. O objetivo deste estudo foi avaliar a DMO em crianças brasileiras portadoras de $\beta$-talassemia maior, comparando-a com grupo controle.

\section{Casuística e Método}

Onze crianças portadoras de TM foram avaliadas (cinco meninos e seis meninas), com mediana de idade de 10,0 anos (variando de 5 a 12 anos). Todos os pacientes estudados estavam em regime transfusional mensal, mantendo hemoglobina pré-transfusional entre 9,5 a 10,5 g/dl desde os primeiros 2 anos de vida. A terapia quelante foi realizada através de administração subcutânea de desferoxamina, na dose de $40 \mathrm{mg} / \mathrm{kg} / \mathrm{dia}$, cinco a seis vezes por semana. Vinte e quatro crianças saudáveis, com mediana de idade de 9,5 anos (variando de 6 a 12 anos), foram incluídas no estudo como grupo controle.

A medida da DMO lombar (L2-L4) foi determinada usando-se emissão dupla de raios-X (Lunar Radiation Corp ${ }^{\circledR}$, Madison, WI, USA) em todos os indivíduos do estudo. Dosagens séricas de fosfatase alcalina e fósforo foram determinadas por método colorimétrico. A concentração de ferritina sérica foi dosada por ensaio imunoenzimático (AbbottLaboratories $\left.{ }^{\circledR}\right)$, e o cálcio ionizado pelo ICA2 Ionized Calcium Analyzer (Radiometer Copenhagen ${ }^{\circledR}$ ). Testes séricos de função hepática, incluindo albumina, tempo de protrombina (método de Quick) e fator V (enzimático, colorimétrico) também foram realizados.
Termo de consentimento livre e esclarecido foi obtido dos responsáveis legais, tanto dos pacientes quanto dos controles. A análise estatística foi realizada por testes não paramétricos. Significância estatística foi atribuída a valores de $\mathrm{p}<0,05$.

\section{Resultados}

Todos os pacientes apresentaram valores normais de cálcio, fósforo, fosfatase alcalina, tempo de protrombina e fator V. O nível de ferritina sérica foi significativamente elevado no grupo de talassêmicos, com mediana de $3.576,0 \mathrm{ng} / \mathrm{ml}$, variando de $2.000,0$ a $6.680,0 \mathrm{ng} / \mathrm{ml}$ (valor normal de 10 a $300 \mathrm{ng} / \mathrm{ml}$ ), e a mediana de hemoglobina pré-transfusional foi de $9,13 \mathrm{~g} / \mathrm{dl}$, variando de 8,0 a $10,3 \mathrm{~g} / \mathrm{dl}$, como pode ser visto na Tabela 1.

Tabela 1. Características laboratoriais de pacientes portadores de beta-talassemia maior

\begin{tabular}{lcc}
\hline \multicolumn{1}{c}{ Parâmetros } & $\begin{array}{c}\text { Valor médio } \\
(\mathrm{DP})\end{array}$ & $\begin{array}{c}\text { Valor de } \\
\text { referência }\end{array}$ \\
\hline $\begin{array}{l}\text { Hemoglobina } \\
\text { pré-transfusional }(\mathrm{g} / \mathrm{dl})\end{array}$ & $9,19( \pm 0,61)$ & 8,5 a $9,5^{\star}$ \\
Ferritina $(\mathrm{ng} / \mathrm{ml})$ & $3832,20( \pm 1449,20)$ & 100 a 300 \\
Albumina $(\mathrm{g} / \mathrm{dl})$ & $4,32( \pm 0,55)$ & 3,2 a 5,6 \\
Atividade de & $81,81( \pm 13,30)$ & $>70 \%$ \\
protrombina $(\%)$ & $1,20( \pm 0,02)$ & 1,14 a 1,29 \\
Cálcio ionizado $(\mathrm{mmol} / \mathrm{l})$ & $4,70( \pm 0,73)$ & 3,5 a 7,0 \\
Fósforo $(\mathrm{mg} / \mathrm{dl})$ & $150,80( \pm 108,25)$ & 38 a 471 \\
Fosfatase alcalina $(\mathrm{U} / \mathrm{l})$ & $75,80( \pm 8,64)$ & 70 a 120 \\
Fator $\mathrm{V}(\%)$ &
\end{tabular}

$\mathrm{DP}=$ desvio padrão; * segundo protocolo tratamento da talassemia maior da Federação Internacional de Talassemia (TIF)

Não foi observada correlação entre DMO e idade dos pacientes, tampouco foi observado diferença dos valores de DMO em relação ao sexo. A perda de massa óssea não apresentou correlação com a hemoglobina pré-transfusional nem com a concentração de ferritina sérica.

O grupo de talassêmicos mostrou peso médio semelhante ao grupo controle $(\mathrm{p}=0,189)$. Com relação à estatura, dois $(18,2 \%)$ pacientes encontravam-se abaixo do percentual 5; quatro $(36,4 \%)$ dentro do percentual 5 ; um $(9,0 \%)$ no percentual 10 e quatro $(36,4 \%)$ entre os percentuais 25 e 75 . A estatura foi significativamente diferente entre os dois grupos estudados, sendo encontrados valores medianos de $124,0 \mathrm{~cm}$ (variando de 107,0 a $150,0 \mathrm{~cm}$ ) no grupo de talassêmicos e 138, $0 \mathrm{~cm}(113,0$ a $151,0 \mathrm{~cm})$ no grupo controle, $\mathrm{p}<0,05$.

Das onze crianças talassêmicas estudadas, sete $(66,3 \%)$ mostraram perda de massa óssea com valor médio de $13,3 \%$ (variando de 10,6\% a 48\%) e três não apresentaram qualquer 


\begin{tabular}{|c|c|c|c|}
\hline \multicolumn{4}{|c|}{$\begin{array}{l}\text { Tabela 2. Características clínicas e } \\
\text { densidade mineral óssea (DMO) de pacientes portadores de } \\
\text { beta-talassemia maior e grupo controle }\end{array}$} \\
\hline Parâmetros & $\begin{array}{c}\text { Pacientes } \\
(\mathrm{n}=11\end{array}$ & $\begin{array}{l}\text { Grupo controle } \\
\quad(n=24)\end{array}$ & $\mathrm{p}$ \\
\hline \multicolumn{4}{|l|}{ Idade (anos) } \\
\hline Mediana & 10,0 & 9,5 & NS \\
\hline Variação & $5-12$ & $6-12$ & \\
\hline Sexo (M/F) & $5 / 6$ & $12 / 12$ & \\
\hline \multicolumn{4}{|l|}{ Peso (kg) } \\
\hline Mediana & 24,0 & 30,5 & NS \\
\hline Variação & $16,0-36,0$ & $21,0-38,0$ & \\
\hline \multicolumn{4}{|l|}{ Altura (cm) } \\
\hline Mediana & 124,0 & 138,0 & $<0,05$ \\
\hline Variação & $(107,0-150,0)$ & $(113,0-151,0)$ & \\
\hline \multicolumn{4}{|l|}{ DMO $(\mathrm{g} / \mathrm{cm}$} \\
\hline Mediana & 0,61 & 0,69 & $<0,05$ \\
\hline Variação & $(0,35-0,76)$ & $(0,58-0,89)$ & \\
\hline
\end{tabular}

perda. Os pacientes mostraram DMO significativamente menor (mediana $0,61 \mathrm{~g} / \mathrm{cm}^{2}$ ) do que os controles (mediana $0,69 \mathrm{~g} / \mathrm{cm}^{2}$ ), como podem ser vistos na Tabela 2 .

\section{Discussão}

Os períodos da infância e adolescência são fundamentais para a aquisição mineral e crescimento ósseo, visto que o pico de massa óssea ocorre até o final da segunda década de vida. O desenvolvimento ósseo pode ser determinado através do tamanho, geometria, qualidade e massa óssea, variáveis influenciadas por fatores genéticos, atividade física, nutrição e hormônios. ${ }^{1,2}$ Crianças com doenças crônicas ou genéticas podem apresentar ganho mineral e crescimento ósseo comprometidos, aumentando o risco de osteoporose precoce. Assim, atualmente, a prevenção de complicações na idade adulta baseia-se em identificar crianças com fragilidade óssea e alto risco de desenvolvimento de osteoporose e fraturas. $^{3,9}$

A talassemia causa anemia crônica e hipóxia com consequente retardo ponderal e puberal. A expansão da medula hematopoética acarreta em erosão do tecido ósseo circunjacente e reabsorção trabecular. ${ }^{4,10}$

A quantificação da doença óssea na talassemia é melhor realizada através da emissão dupla de raio X, e a redução da DMO é o maior preditor de risco de fraturas. Esta é determinada pelo pico de massa óssea obtido durante a adolescência e a taxa de perda óssea após esta fase..$^{5-7,10}$

Este é o primeiro trabalho, descrito em literatura, que determinou a perda de massa óssea em crianças talassêmicas no Brasil. No presente estudo, a reduzida DMO, observada em $72 \%$ dos pacientes, é similar à observada em estudos prévios. $^{5-7,10}$
É considerado que o tratamento adequado destes pacientes, com a combinação de transfusão e quelação de ferro, seja capaz de reduzir as taxas de fratura e aumentar a massa óssea. ${ }^{6,7}$ Neste trabalho, entretanto, foi observada perda óssea em indivíduos talassêmicos independente destas terapêuticas. Pacientes com TM sofrem contínua absorção óssea, cuja morbidade é fortemente correlacionada com o fenótipo hematológico e a presença de hipogonadismo. Levando-se em consideração que a formação óssea é contínua, terapêuticas baseadas na suplementação de cálcio, reposição hormonal, bisfosfonatos e fatores de crescimento poderão contribuir positivamente durante a vida destes pacientes, evitando complicações ósseas. ${ }^{8}$

Em resumo, os dados obtidos neste trabalho confirmam relevante perda óssea na maioria das crianças com TM. Este achado reforça a necessidade de identificação e tratamento adequado da osteopenia nestes pacientes, contribuindo na diminuição da morbidade destes indivíduos.

\section{Abstract}

Osteoporosis is characterized by low bone mass and disruption of bone architecture, resulting in greater bone fragility with increased risk of fractures. Bone disease is an important cause of morbidity in beta thalassemia major patients. Osteoporosis has been described extensively in adult thalassemia. However, there are no studies describing Brazilian thalassemic children. We evaluated eleven patients with beta thalassemia major (median age of 10.0 years, range from 5 to 12 years) and twenty-four healthy children (median age of 9.5 years, range from 6 to 12 years), using dual $X$-ray absorptiometry to assess bone mineral density (BMD). Analysis of biochemical markers such as serum ferritin concentration, ionized calcium, alkaline phosphatase, phosphorus, albumin, prothrombin time and factor $V$ was performed. The height was very different between the groups, $p<0.05$. The thalassemic patients showed significantly lower $B M D$ (median $0.61 \mathrm{~g} / \mathrm{cm}^{2}$ ) than control subjects (median $0.69 \mathrm{~g} / \mathrm{cm}^{2}$ ) $-p<0.05$. The relevant bone loss in the majority of thalassemic children studied emphasizes the need for identification and appropriate treatment of osteopenia, thereby reducing the morbidity of these patients. This is the first study described in the literature that determined bone mineral loss in Brazilian thalassemic children. Rev. Bras. Hematol. Hemoter. 2008; 30(6): 445-448.

Key words: Thalassemia; bone mineral density; osteopenia; densitometry; anemia.

\section{Referências Bibliográficas}

1. The WHO Study Group. Assessment of fracture risk and its application to screening for postmenopausal osteoporosis. Switzerland:World Health Organization; 1994.

2. NIH Consensus Statement, n.111. Osteoporosis prevention, diagnosis and therapy. 2000;17:1-36.

3. Campos LMA, Liphaus BL, Silva CAA, Pereira RMR. Osteoporose na infância e na adolescência. J Pediatr 2003;79:481-8.

4. Wonke B. Bone disease beta thalassaemia major. Br J Haematol. 1998;103:897-901. 
5. Voskaridou E, Kyrtsonis MC, Terpos E, et al. Bone resorption is increased in young adults with thalassaemia major. Br J Haematol. 2001;112:36-41.

6. Mahachoklertwattana P, Pootrakul P, Chuansumrit A, Choubtum L, Sriphrapradang A, Sirisriro R, et al. Association between bone mineral density and erythropoiesis in Thai children and adolescents with thalassemia syndromes. J Bone Miner Metab. 2006;24(2): 146-52.

7. Dresner Pollack R, Rachmilewitz E, Blumenfeld A, Idelson M, Goldfarb AW. Bone mineral metabolism in adults with betathalassaemia major and intermedia. Br J Haematol. 2000;111(3): 902-7.

8. Voskaridou E, Anagnostopoulos A, Konstantopoulos K, Stoupa E, Spyropoulou E, Kiamouris C, et al. Zoledronic acid for the treatment of osteoporosis in patients with beta-thalassemia: results from a single-center, randomized, placebo-controlled trial. Haematologica. 2006;91(9):1193-202.

9. Bachrach LK. Consensus and controversy regarding osteoporosis in the pediatric population. Endocr Pract. 2007;13(5):513-20.

10. Karimi M, Ghiam AF, Hashemi A, Alinejad S, Soweid M, Kashef S. Bone mineral density in beta-thalassemia major and intermedia. Indian Pediatr. 2007;44(1):29-32.

Avaliação: Editor e dois revisores externos

Conflito de interesse: não declarado

Recebido: 07/04/2008

Aceito após modificações: 31/05/2008 